\title{
Sharing of ADHD Information between Parents and Teachers Using an EHR-Linked Application
}

Jeremy J. Michel ${ }^{1,2}$ Stephanie Mayne ${ }^{3,4}$ Robert W. Grundmeier ${ }^{1,2}$ James P. Guevara 1,4,5

Nathan J. Blum ${ }^{6}$ Thomas J. Power ${ }^{7}$ Emily Coffin ${ }^{8}$ Jeffrey M. Miller ${ }^{2}$ Alexander G. Fiks $1,2,3$

\footnotetext{
${ }^{1}$ Department of Pediatrics, Perelman School of Medicine, University of Pennsylvania, Philadelphia, Pennsylvania, United States

${ }^{2}$ Department of Biomedical and Health Informatics, The Children's Hospital of Philadelphia, Philadelphia, Pennsylvania, United States

${ }^{3}$ Center for Pediatric Clinical Effectiveness, The Children's Hospital of Philadelphia, Philadelphia, Pennsylvania, United States

4 PolicyLab: Center to Bridge Research, Practice, and Policy, The Children's Hospital of Philadelphia, Philadelphia, Pennsylvania, United States

${ }^{5}$ Leonard Davis Institute of Health Economics, University of Pennsylvania, Philadelphia, Pennsylvania, United States

6 Division of Developmental and Behavioral Pediatrics, The Children's Hospital of Philadelphia, Philadelphia, Pennsylvania, United States

7 Department of Child and Adolescent Psychiatry and Behavioral Sciences, The Children's Hospital of Philadelphia, Philadelphia, Pennsylvania, United States

${ }^{8}$ The Children's Hospital of Philadelphia, Philadelphia, Pennsylvania, United States
}

Address for correspondence Jeremy J. Michel, MD, MHS, Department of Biomedical and Health Informatics, The Children's Hospital of Philadelphia, 2716 South Street, Philadelphia, PA 19146, United States (e-mail: michelj@email.chop.edu).

Appl Clin Inform 2018;9:892-904.

\section{Abstract}

Keywords

- attention-deficit/ hyperactivity disorder

- information dissemination

- surveys and questionnaires

- communications

- other clinical informatics applications
Background Appropriate management of attention-deficit/hyperactivity disorder (ADHD) involves parents, clinicians, and teachers. Fragmentation of interventions between different settings can lead to suboptimal care and outcomes. Electronic systems can bridge gaps across settings. Our institution developed an email-based software to collect ADHD information from parents and teachers, which delivered data directly to the clinician within the electronic health record (EHR).

Objective We sought to adapt our institution's existing EHR-linked system for ADHD symptom monitoring to support communication between parents and teachers and then to assess child characteristics associated with sharing of ADHD information.

Methods We updated our software to support automated sharing of ADHD information between parents and teachers. Sharing was optional for parents but obligatory for teachers. We conducted a retrospective cohort study involving 590 patients at 31 primary care sites to evaluate a system for sharing of ADHD-specific health information between parents and teachers. We used multivariable logistic regression to estimate associations between child characteristics and parental sharing. We further investigated the association between child characteristics and viewing of survey results delivered through the electronic communication system.

Results Most parents (64\%) elected to share survey results with teachers at the first opportunity and the vast majority (80\%) elected to share all possible information. received

September 4, 2018

accepted after revision

October 20, 2018 (c) 2018 Georg Thieme Verlag KG Stuttgart · New York
DOI https://doi.org/

10.1055/s-0038-1676087.

ISSN 1869-0327. 
Parents who elected to share usually continue sharing at subsequent opportunities (89\%). Younger child age and performance impairments were associated with increased likelihood of sharing. However, parents viewed only $16 \%$ of teacher submitted surveys and teachers only viewed $30 \%$ of surveys shared by parents.

Conclusion This study demonstrates that electronic systems to capture ADHD information from parents and teachers can be adapted to support communication between them, and that parents are amenable to sharing ADHD information with teachers. However, strategies are needed to encourage viewing of shared information.

\section{Background and Significance}

Attention-deficit/hyperactivity disorder (ADHD) is the most common inheritable chronic childhood neurobehavioral disorder, affecting approximately $6.5 \%$ of school-aged children. ${ }^{1-3}$ The effects of ADHD manifest in multiple environments, but most commonly lead to issues at both home and school. ${ }^{2}$ As a result, effective management of children with ADHD involves collaboration between parents, clinicians, and teachers. ADHD rating scales (e.g., Vanderbilt scales) are the standard method for clinicians to collect data and monitor disease severity during evaluation and treatment of ADHD. ${ }^{2,4}$ In clinical practice, rating scales represent a one-way, parallel communication from parent and teacher to clinician; there is a missed opportunity to foster communication between parents and teachers, key members of a child's ADHD management team.

Recognizing the need for collaboration, the 2011 American Academy of Pediatrics Guidelines for the care of children with ADHD stress the need for shared decision making (SDM). ${ }^{2}$ SDM involves the identification of possible treatment options, sharing of preferences and goals for intervention, and implementation of the preferred treatment plan. ${ }^{5}$ Although research has begun to demonstrate benefits of SDM in ADHD care, ${ }^{6,7}$ communication challenges between families, clinicians, and schools remain. Standard use of data collection instruments does not support SDM as parents and teachers do not have access to data collected by clinicians. The role of technology in facilitating this information sharing is under investigation. ${ }^{8}$

Despite the need for teamwork across settings, fragmentation of interventions between schools and the health care system often undermines ADHD care and limits SDM. ${ }^{9-12}$ Parents and teachers may wish to collaboratively set behavior and education goals informed by areas of concern identified during ADHD evaluations. In addition, through sharing parents may become aware of issues that present exclusively at school that should be addressed in treatment plans. When families experience difficulty communicating their preferences and goals for treatment to providers and educators involved in their child's care, the quality and adherence to treatment plans may be adversely impacted. ${ }^{13}$ Despite ubiquitous mobile devices transforming interpersonal communication, ${ }^{14}$ sharing of ADHD information between interested parties continues to be inconsistent. ${ }^{15,16}$ Multiple barriers, including school policies, can lead to decreased communication between parents, teachers, and clinicians. ${ }^{17-20}$ While validated rating scales have helped clinicians diagnose and monitor ADHD, ${ }^{4,21,22}$ less work has been done to address barriers to communication and fragmentation of care, which can result in suboptimal outcomes. ${ }^{15-17}$ In addition, poor communication and coordination may result in duplication of services and inadequate monitoring and tailoring of treatment to align with a child's needs and his or her family's preferences and goals. ${ }^{23,24}$ Quality of care and uptake of evidence-based therapies have been reported to be low in poorly integrated systems. ${ }^{9,20,25}$ Informatics-based interventions that can facilitate cross-systems collaboration and support ongoing communication are urgently needed. ${ }^{16}$

Building on prior success in using an internally developed tool for ADHD monitoring by clinicians, ${ }^{26-30}$ we sought to develop a system to improve communication between parents and teachers to decrease fragmentation of care. We targeted communication between parents and teachers due to recognition that this data exchange channel was unsupported by our electronic health record (EHR), and hypothesized that our current clinical decision support (CDS) could be modified to support this information sharing. During this study, we evaluated patient characteristics that may have influenced parents and teachers to use this system to share and view patient ADHD information. Recently, it has been demonstrated that Web-based portals help improve coordination among parents and providers for the management of pediatric chronic medical conditions. ${ }^{31,32}$ Web-based interventions have been developed for ADHD as well, and have been shown to improve rating scale completion and patient satisfaction and patient outcomes. ${ }^{26,33-37}$ Although portals have been developed for pediatricians to collect ADHD information from parents and teachers and can support communication, no previous interventions support automated sharing of ADHD survey responses between parents and teachers. Based on studies of children with other chronic medical conditions, ${ }^{31,32}$ we hypothesized that parents of more severely affected children would be more likely to share information with teachers.

\section{Objective}

We sought to adapt our institution's EHR-linked system for ADHD symptom monitoring to support communication between parents and teachers. Upon completion, we then evaluated child characteristics associated with sharing of ADHD information to determine the relationship between socioeconomic and other clinical factors on willingness to share information. 


\section{Methods}

\section{Study Setting and Population}

We conducted this study within the Children's Hospital of Philadelphia (CHOP) ambulatory care network. We undertook a retrospective cohort study to evaluate the use of a system, the ADHD Care Assistant, to facilitate sharing of information on ADHD symptoms, performance impairments, medication side effects, comorbid symptoms, and treatment goals between parents and teachers. The original software was made available on November 1, 2014. The study period began on January 25, 2017 when the updates to the software were implemented to allow for sharing between parents and teachers and ended on June 16, 2017 to align with the end of the school year. In our analysis, we included all patients whose parent/guardian used the ADHD Care Assistant at least once during the study period $(N=590)$. A study flow diagram ( $>$ Fig. 1) demonstrates how the study sample was derived. The ADHD Care Assistant was displayed to clinicians for all patients with a diagnosis of ADHD or a chief complaint of "behavior problem" within the reason for visit select box. Use of this system was optional and offering it to families was solely at the discretion of the primary clinician. The institutional review board at CHOP approved this project and granted a waiver of consent.

\section{The ADHD Care Assistant}

The ADHD Care Assistant ( - Fig. 2) is an internally developed CDS module used across the CHOP's network of 31 primary care sites to improve data collection from parents and teachers of school-aged children with ADHD. ${ }^{26}$ Through the ADHD Care Assistant, a clinician can register a parent and teacher to receive scheduled ADHD symptom surveys, which are delivered directly to the parent's personal email address. A form given to the teacher provides instructions on how to register and receive surveys, which are then delivered directly to the teacher's email address. Parents and teachers completed surveys by clicking the survey-specific link and assessing information on symptoms, performance impairments, comorbid symptoms, and medication side effects based on the Vanderbilt ADHD Rating Scales. ${ }^{4,21}$ In addition, parents provided information on their ADHD treatment preferences and goals using the ADHD Preference and Goal Instrument (PGI). ${ }^{6,38}$ Both the Vanderbilt and PGI are validated survey instruments for children with ADHD. The initial parent survey instrument contained 104 questions while the initial teacher survey and follow-up survey instruments each contained approximately 45 questions. Prior to initializing emailed surveys, a clinician obtains a Health Insurance Portability and Accountability Act (HIPAA) release in the office and gave the parent a Family Educational Rights and Privacy Act (FERPA) release form so that the school could document parental permission for the teacher to use the system.

Our institution uses this system for children with behavior concerns as a screening tool and to track symptom severity, performance, treatment goals, and, when applicable, medication side effects for patients diagnosed with ADHD. No particular script was provided to help clinicians introduce the

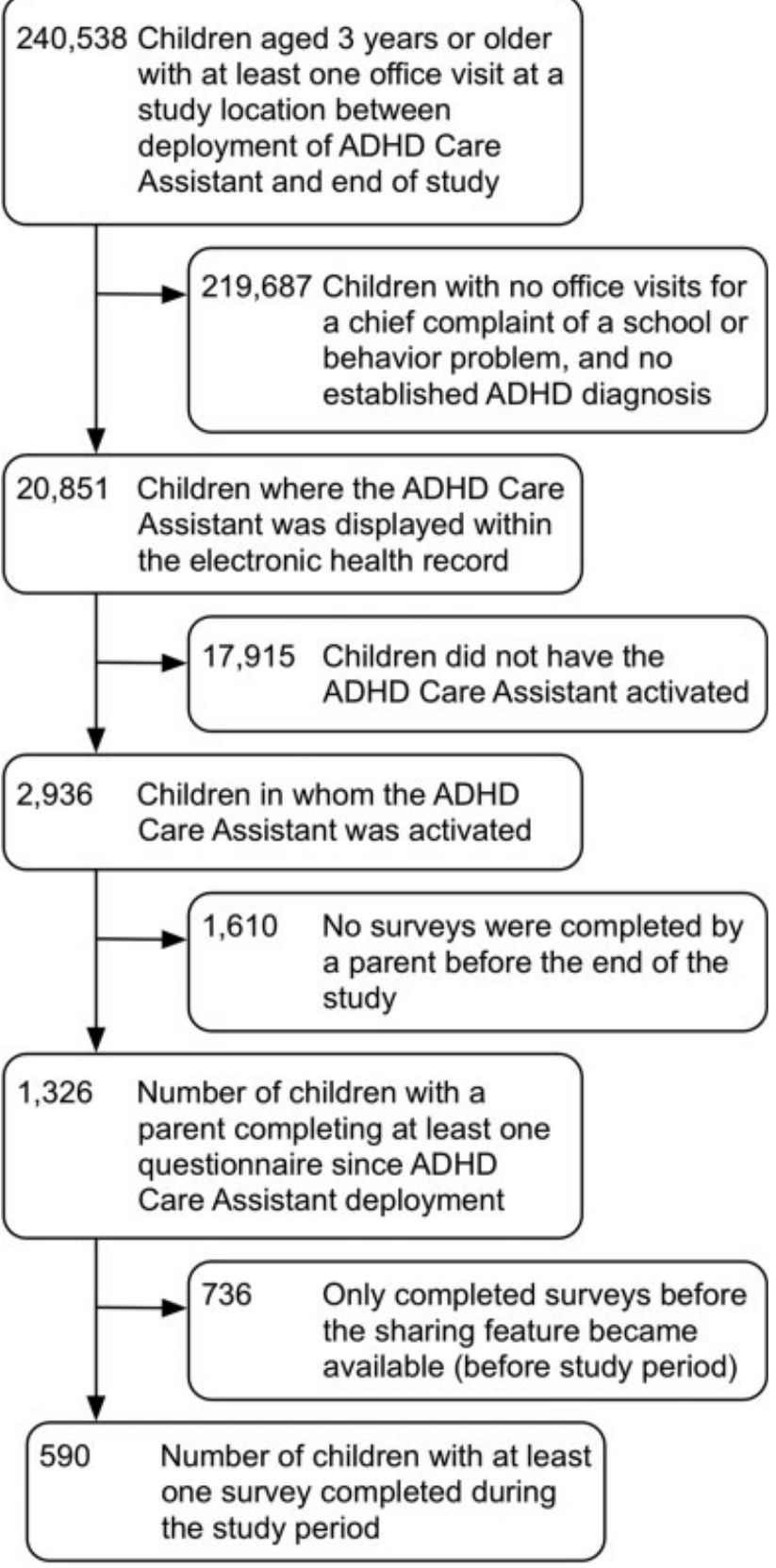

Fig. 1 Study flow diagram. Approximately $8.7 \%$ of patients seen since the introduction of the attention-deficit/hyperactivity disorder

(ADHD) Care Assistant have ADHD or had a concern raised for ADHD. Of these, $14 \%$ were registered by clinicians to use the intervention. Nearly half (45\%) of parents who registered to use the intervention completed at least one survey. Of parents who used the system, $44 \%$ completed at least one survey during the study period.

system. Use of the system was voluntary and activation of the system required a primary care clinical encounter. In this system, parents annually updated their child's teacher. Consistent with standard practice, we initially developed this system with a unidirectional data flow from parents and teachers directly into the EHR for clinician review and did not facilitate parent-teacher communication. To inform development of the system to support parent-teacher communication, we solicited input from 8 parents, 11 pediatricians, and 8 educators in a series of stakeholder meetings between September 2015 and 


\begin{tabular}{|l|l|}
\hline ADHD Assistant \\
\end{tabular}

Fig. 2 The Attention-Deficit/Hyperactivity Disorder (ADHD) Care Assistant. The ADHD Care Assistant has these functions: (1) email-based support for gathering information from parents and teachers including ADHD symptoms, performance impairments, and treatment preferences and goals, (2) automatic delivery of information to clinicians through the electronic health record (EHR), and (3) links to educational materials.

September 2016. Based on stakeholder input, we enhanced the system to support parents selectively sharing portions from each submitted survey with a teacher, a key feature not present in similar software systems, and one that had yet to be studied. Secure viewing of submitted surveys was achieved using Cisco Registered Envelope Service (Cisco System, Inc., San Jose, California, United States), which requires users to register prior to accessing secured messages. ${ }^{39}$ The teacher-parent information sharing functionality is shown in Fig. 3 .

\section{Endpoints}

We selected as the primary study endpoint parent/guardian sharing of any component of the parent ADHD survey at first opportunity during the study period. When sharing occurred, we further analyzed the type of information shared (symptoms, performance, goals, and/or medication side effects). For patients with multiple opportunities to share, we conducted a secondary analysis to assess patterns in sharing over the study period. For this secondary analysis, we restricted the sample to children whose parent/guardian shared at the first opportunity and completed at least two surveys during the study period. Finally, we investigated the frequency with which teachers and parents viewed shared information. For this analysis, we included all surveys completed during the study period for our study population.

Child Characteristics Associated with Parent Sharing We assessed child characteristics that were potential drivers of parent/guardian sharing. Characteristics included ADHD symptom and performance scores calculated using standard Vanderbilt Rating Scale scoring practices. ${ }^{4}$ Specific characteristics used in the analysis included: (1) ADHD symptoms (categorized as no symptoms, inattentiveness only, hyperactivity only, or combined symptoms), (2) performance impairment (categorized as no impairments, academic impairment only, interpersonal impairment only, or both), (3) symptoms of comorbidities (none, any, and symptoms for oppositional defiance disorder, conduct disorder, or anxiety/depression), (4) prior diagnosis or 


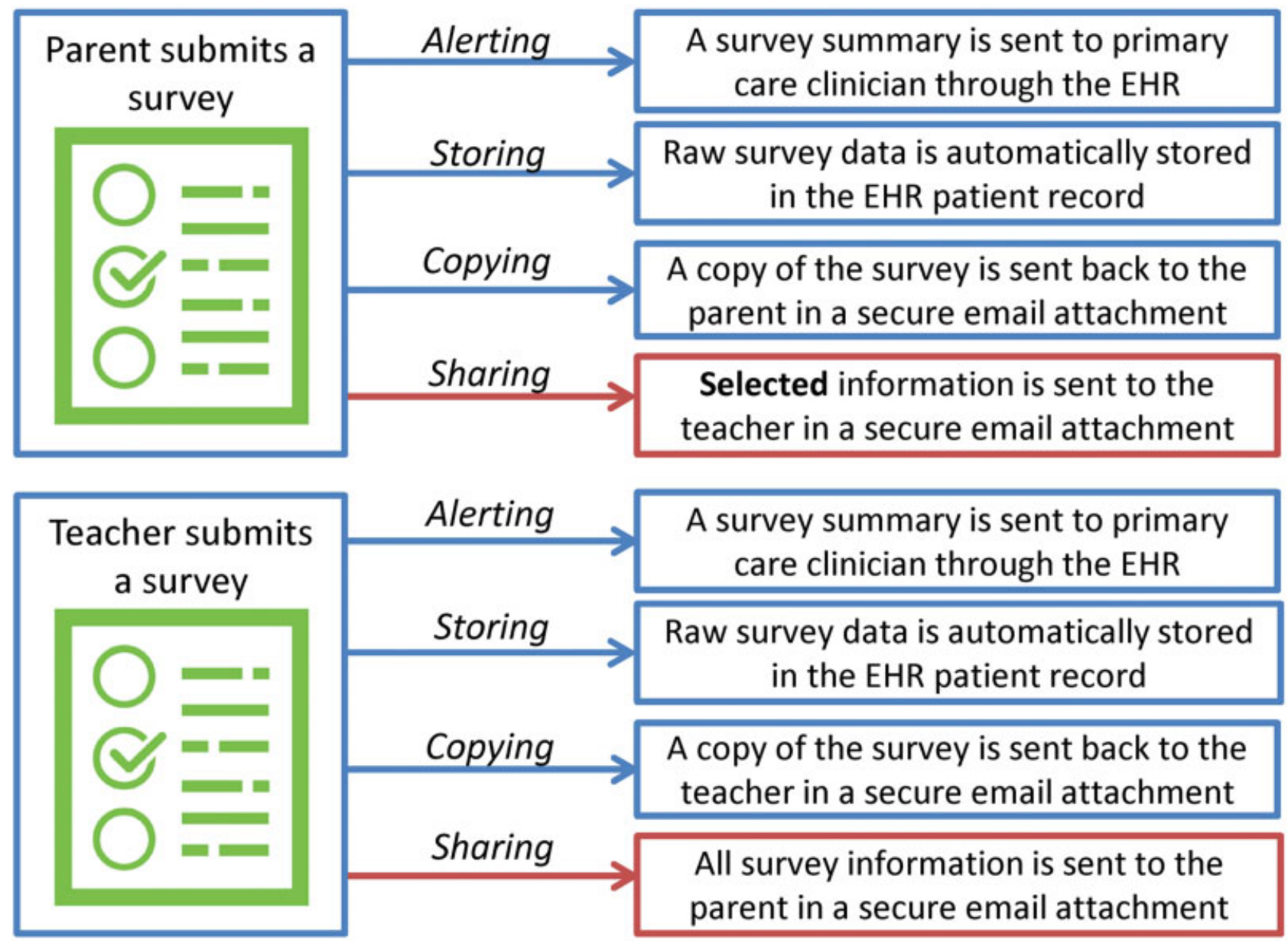

Fig. 3 Messages handling upon survey completion. Upon survey submission, the clinician is alerted, the raw data are stored in the electronic health record (EHR), and a copy is sent to the survey submitter. Parent survey data can be selectively shared with teachers and teacher survey data are automatically shared with parents.

problem list entry of ADHD, (5) medication side effects (categorized as none, mild, or moderate/severe side effects), (6) survey type (initial or follow-up), and (7) completion of a teacher survey during the study period. In our primary analysis, we included each family's first survey during the study period. That first survey could be either an initial or follow-up ADHD rating scale survey depending on whether it was the parent's first time using the ADHD Care Assistant rating scale collection system (initial survey) or whether they were already using the system at the start of the study (follow-up survey). While the initial and follow-up survey both have sections addressing symptom and performance impairment, it is important to note that only the initial survey contained the treatment goal section and only the follow-up survey contained the medication side effect section. Parent, clinician, and teacher demographics were not obtained as part of this study as we relied on data collected during the provision of routine medical care.

\section{Covariates}

Covariates included patient age (3-12 vs. 13-18 years), sex, race (white, African American, or other race), Hispanic/ Latino ethnicity, insurance status (Medicaid vs. private), practice setting (urban vs. suburban), ADHD medication orders, and parent report of ADHD medication use. We selected these age ranges to evaluate differences in sharing in primary school versus high school. Ten parents of preschool-aged children used the system to submit ADHD information from parents and preschool educators. We performed a subgroup analysis on the 9- to 12-year-old cohort to assess if this population was significantly different from the younger primary school group. If either a parent reported ADHD medication use on the survey or the EHR contained an order for ADHD medications, this patient was classified as a medication user.

\section{Statistical Analysis}

We examined the distribution of ADHD characteristics and patient demographics in the study population. For our primary analysis, we examined bivariate associations between these characteristics and whether parents decided to share information at the first opportunity using chisquared tests. After identifying patient ADHD characteristics associated with sharing (at the $p<0.1$ level), we used multivariable logistic regression to estimate associations of these characteristics with sharing at the first opportunity. Characteristics examined in the full model included ADHD 
symptoms, performance impairments, survey type, and teacher survey completion. We controlled for patient covariates that were associated with sharing in bivariate analyses, including age, race, insurance status, and ADHD medication use based on documentation in either the EHR or parent report through the ADHD Care Assistant.

We then examined viewing patterns of survey results by parents and teachers. We calculated the proportions of shared parent surveys that were viewed by the teacher and teacher surveys that were viewed by the parent (all teacher surveys are shared automatically). We looked for associations between patient characteristics and teacher viewing of surveys. Finally, we examined patterns of sharing over time. We calculated the proportion of children whose parent/guardian shared at least two surveys, and examined the distribution of total number of surveys shared by each parent. We examined whether there were differences in parent and teacher viewing of survey results between parents who chose to share a subsequent survey and those who did not.

\section{Results}

\section{Study Population}

Between January 25, 2017 and June 16, 2017, 590 parents/ guardians completed at least one survey using the ADHD Care Assistant for their child; 232 of these children had a teacher survey completed during the study period (39.3\%). A total of 1,006 parent surveys and 493 teacher surveys were completed overall (range: 1-10 parent surveys per child and 0-10 teacher surveys per child).

-Table 1 presents the distribution of demographic characteristics of the study population. Most children (88\%) in the study had a diagnosis of ADHD either before or during the study period. Overall, $85.9 \%$ of patients were aged 3 to 12 years, $70.2 \%$ were male. Note that $48.3 \%$ were white, 40.0\% were African American, and 5.1\% were Hispanic/ Latino. A total of $63.6 \%$ were privately insured, and $61.5 \%$ were from suburban practices. ADHD medication use was documented and/or reported for $87 \%$ of children.

Value of Sharing to Parent and Teacher Stakeholders During the preimplementation stakeholder meetings, parents and teachers both indicated desire for access to the information they personally submitted and to the information that was submitted by their counterparts. Stakeholder parents expressed concern that some parents may wish to control which information was shared with the teacher, using examples from the Vanderbilt Rating Scale of answers to particular questions that people may not like to share (e.g., \#32: Has stolen things that have value). ${ }^{21}$ To address this acceptability concern, we developed our system to allow selective sharing at the section level. The shareable sections were ADHD symptoms, performance impairments, treatment goals (initial survey only), and medication side effects (follow-up surveys only).

Parents and teachers both requested to see "what the clinician sees" in terms of presentation of results. To support this function, we provided both a longitudinal view and raw
Table 1 Demographic and clinical characteristics of study population and bivariate associations with whether parents chose to share ADHD survey information with teachers on the first survey in the study period

\begin{tabular}{|c|c|c|c|}
\hline $\begin{array}{l}\text { Demographic } \\
\text { characteristic }\end{array}$ & $\begin{array}{l}N(\%) \text { with } \\
\text { characteristic } \\
\text { (column \%) }\end{array}$ & $\begin{array}{l}\text { Shared at first } \\
\text { opportunity }-N \\
\text { (row \%) }\end{array}$ & $p$-Value ${ }^{c}$ \\
\hline Total, N & 590 & $380(64.4)$ & \\
\hline Age $(y)$ & & & $<0.001$ \\
\hline $3-12$ & 507 (85.9) & $345(68.1)$ & \\
\hline $13-18$ & $83(14.1)$ & $35(42.2)$ & \\
\hline Sex & & & 0.8 \\
\hline Male & $414(70.2)$ & $268(64.7)$ & \\
\hline Female & $176(29.8)$ & $112(63.6)$ & \\
\hline Race & & & 0.03 \\
\hline White & $285(48.3)$ & 169 (59.3) & \\
\hline $\begin{array}{l}\text { African } \\
\text { American }\end{array}$ & $236(40.0)$ & $160(67.8)$ & \\
\hline Other race & 69 (11.7) & $51(73.9)$ & \\
\hline Ethnicity & & & 0.3 \\
\hline $\begin{array}{l}\text { Hispanic/ } \\
\text { Latino }\end{array}$ & $30(5.1)$ & $22(73.3)$ & \\
\hline $\begin{array}{l}\text { Not Hispanic/ } \\
\text { Latino }\end{array}$ & 560 (94.9) & 358 (63.9) & \\
\hline $\begin{array}{l}\text { Insurance } \\
\text { status }\end{array}$ & & & 0.06 \\
\hline Medicaid & $215(36.4)$ & $149(69.3)$ & \\
\hline Private & $375(63.6)$ & 231 (61.6) & \\
\hline $\begin{array}{l}\text { Primary care } \\
\text { practice type }\end{array}$ & & & 0.1 \\
\hline Urban & $227(38.5)$ & $155(68.3)$ & \\
\hline Suburban & $363(61.5)$ & $225(62.0)$ & \\
\hline $\begin{array}{l}\text { ADHD } \\
\text { medication use }{ }^{b}\end{array}$ & & & $<0.001$ \\
\hline Yes & 513 (86.9) & $314(61.2)$ & \\
\hline No & $77(13.1)$ & $66(85.7)$ & \\
\hline
\end{tabular}

Abbreviations: ADHD, attention-deficit/hyperactivity disorder; EHR, electronic health record.

a Parent and teacher surveys were administered at regular intervals set by the child's primary care clinician. In our primary analysis, we included each family's first survey during the study period and determined whether the parent chose to share any component of the survey with their child's teacher. based on EHR documentation and parent report. Of children with medications listed on medication recorded in the EHR, 92.0\% were on a stimulant only, $4.7 \%$ were on a stimulant and either an $\alpha$ agonist or atomoxetine, $1 \%$ were on an $\alpha$ agonist only, and $<1 \%$ were on other combinations. ${ }^{c} p$-Values calculated using chi-squared tests to compare the proportion of parents in each category that chose to share information with teachers at the first opportunity.

data within the reports sent to parents and teachers. We used the same color-coding of results that was provided to clinicians-green for good, yellow for borderline, and red for areas of concern. We did not provide scores or cutoffs for diagnosis, as this was felt to be beyond the reach of the CDS 


\title{
To the parent or guardian of
}

\author{
Thank you for completing a survey through CHOP's ADHD Care Assistant. Please see below \\ for a summary of your results.
}

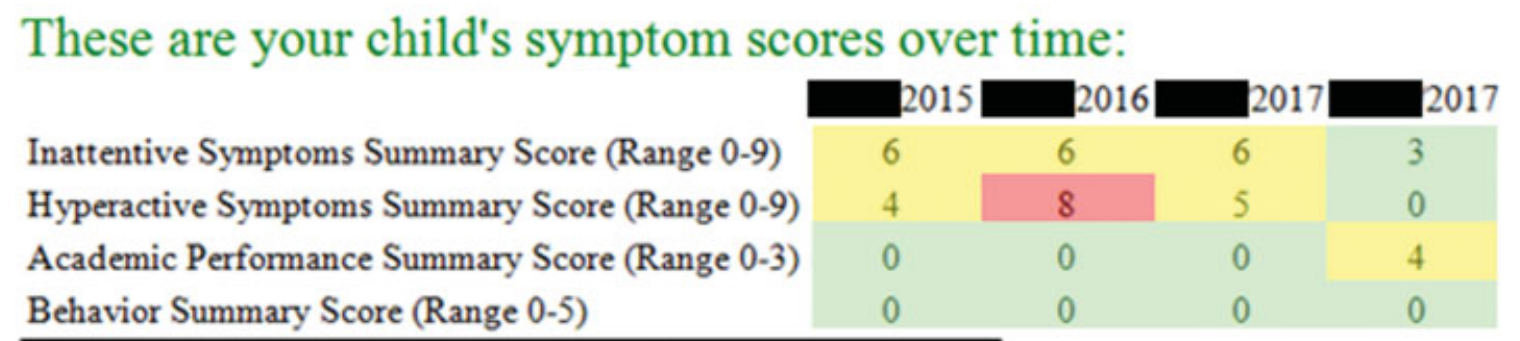

Quick guide to interpreting ADHD Summary Scores (Above):
Higher numbers generally mean more symptoms.
Symptoms Acceptable (Green)
Symptoms Borderline (Yellow)
Symptoms Conceming (Red)

These are the responses that were just submitted: ( Inattentive Symptoms

\section{Does not pay attention to details or makes careless mistakes with, for example, homework: Often Has difficulty keeping attention to what needs to be done: Often \\ Does not seem to listen when spoken to directly: Often \\ Does not follow through when given directions and fails to finish activities (not due to refusal or failure to \\ 1. ก $\cap \Omega$}

Fig. 4 Example of emailed report sheet. This example of a patient-directed report sheet generated by the Attention-Deficit/Hyperactivity Disorder (ADHD) Care Assistant includes longitudinal data summarizing all scores submitted since the patient was registered and includes the questions/answers submitted on the most recent ADHD survey response. Parent and teachers reports are formatted the same.

application and would not facilitate communication between parents and teachers.

Parent and teacher stakeholders reviewed prototype reports and provided feedback during the iterative development process. The stakeholders indicated that the layout and content of the result reports in their final form would be beneficial in supporting SDM surrounding ADHD diagnosis and management ( - Fig.4). Overall, stakeholders valued the possibility of an electronic tool to address known difficulties in communication of ADHD information.

\section{Sharing at the First Opportunity}

Out of 590 patients whose parent completed a survey during the study period, 380 parents chose to share results with their child's teacher at the first opportunity (64.4\%, - Table 1). Most parents $(304,80.0 \%)$ shared all sharable survey components with their child's teacher. In bivariate analysis, parents of children aged 3 to 12 and of children who were publically insured were more likely to share than parents of adolescents or parents of privately insured children ( - Table 1 ). In a subgroup analysis, we noted no difference between the youngest (3-8 years) and middle aged (9-12 years) patient groups. Parents of white children were less likely to share, as were parents of children using medication for ADHD. Practice location (urban vs. suburban) did not affect the likelihood of decision to share.
In bivariate analyses ( - Table 2), parents were more likely to share an initial compared with a follow-up survey $(p<0.001)$. In addition, parents whose child was manifesting elevations in ADHD symptoms, performance impairments, or symptoms of any comorbidity (scoring as per Vanderbilt Rating Scale instructions) were significantly more likely to share information with teachers ( $p<0.05$ for all comparisons). Having no prior diagnosis of ADHD was associated with an increased likelihood of a parent selecting to share the results with the teacher. Medication side effects were not associated with sharing. Parents of children whose teacher completed a survey during the follow-up period were also more likely to share information with teachers ( $p=0.001)$. In a secondary analysis, we looked at the relationship between parental preferences and goals and information sharing. We found that these were not associated ( $p \geq 0.05$ for all preference and goal categories).

In multivariable models controlling for patient age, race, insurance status, and ADHD medication use, parents of children experiencing ADHD-related performance impairments in academics, interpersonal relationships, or both were significantly more likely to share relative to those with no impairments (odds ratios [ORs] of 1.95 [95\% confidence interval [CI]: 1.21, 3.15], 2.03 [1.09, 3.76], and 2.09 [1.26, 3.44], respectively, - Table 3 ). In addition, parents of children whose teacher had completed a survey during the 
Table 2 Bivariate associations of ADHD characteristics with parents' decision to share information with teachers at the first opportunity

\begin{tabular}{|c|c|c|c|}
\hline ADHD characteristics: Parent surveys ${ }^{a}$ & $\begin{array}{l}N(\%) \text { with characteristic } \\
\text { (column \%) }\end{array}$ & $\begin{array}{l}\text { Shared at first opportunity - } \\
N \text { (row \%) }\end{array}$ & $p$-Value ${ }^{c}$ \\
\hline Total, $N$ & 590 & $380(64.4)$ & \\
\hline Survey type & & & $<0.001$ \\
\hline Initial & $180(30.5)$ & $141(78.3)$ & \\
\hline Follow-up & $410(69.5)$ & $239(58.3)$ & \\
\hline Teacher completed survey during study & & & 0.001 \\
\hline Yes & $232(39.3)$ & $168(72.4)$ & \\
\hline No & $358(60.7)$ & $212(59.2)$ & \\
\hline ADHD diagnosis or problem list entry & & & $<0.001$ \\
\hline Yes & $520(88.1)$ & $325(62.5)$ & \\
\hline No & $70(11.9)$ & $55(78.6)$ & \\
\hline ADHD symptoms reported ${ }^{\mathrm{b}}$ & & & 0.02 \\
\hline No ADHD symptom criteria met & $302(51.2)$ & $176(58.3)$ & \\
\hline Inattentive symptom criteria met & $124(21.0)$ & $89(71.8)$ & \\
\hline Hyperactive symptom criteria met & $31(5.3)$ & $21(67.7)$ & \\
\hline $\begin{array}{l}\text { Both inattentive and hyperactive } \\
\text { symptom criteria met }\end{array}$ & $133(22.5)$ & $94(70.7)$ & \\
\hline Performance impairments ${ }^{\mathrm{b}}$ & & & $<0.001$ \\
\hline No impairment criteria met & $184(31.2)$ & $95(51.6)$ & \\
\hline Academic only & $163(27.6)$ & $115(70.6)$ & \\
\hline Interpersonal only & $69(11.7)$ & $47(68.1)$ & \\
\hline Both academic and interpersonal & $174(29.5)$ & $123(70.7)$ & \\
\hline Symptoms of comorbidities ${ }^{\mathrm{d}}(N=180)$ & & & 0.03 \\
\hline No criteria for comorbidities met ${ }^{\mathrm{b}}$ & $102(56.7)$ & $74(72.6)$ & \\
\hline Criteria met for 1 or more comorbidity & $78(43.3)$ & $67(85.9)$ & \\
\hline \multicolumn{4}{|l|}{ Criteria met for specific comorbidities } \\
\hline Oppositional defiance & $66(36.7)$ & $55(83.3)$ & 0.2 \\
\hline Conduct disorder & $12(6.7)$ & $10(83.3)$ & 0.7 \\
\hline Anxiety or depression & $37(20.6)$ & $34(91.9)$ & 0.03 \\
\hline Medication side effects $(N=410)$ & & & 0.8 \\
\hline No side effects & $46(11.2)$ & $25(54.4)$ & \\
\hline Mild side effects & $119(29.0)$ & $69(58.0)$ & \\
\hline Moderate/severe side effects & $245(59.8)$ & $145(59.2)$ & \\
\hline
\end{tabular}

Abbreviation: ADHD, attention-deficit/hyperactivity disorder.

${ }^{a} A D H D$ symptoms and performance items were asked on both initial and follow-up surveys ( $N=590$ parent surveys and 232 teacher surveys). Comorbidities were only assessed on initial surveys $(N=180$ parent surveys and 104 teacher surveys), while side effects were only assessed on follow-up surveys ( $N=410$ parent surveys and 128 teacher surveys).

bscoring performed according to instructions provided in the Vanderbilt survey handout. No symptoms/evidence in each category indicates that a parent or teacher did not indicate a positive in any subsection of the scale. Inattentive and hyperactive symptoms require 6/9 positive responses. Other sections of the Vanderbilt had varying criterion for positives.

${ }^{c} p$-Values calculated using chi-squared tests to compare the proportion of parents in each category that chose to share information with teachers at the first opportunity.

${ }^{\mathrm{d}}$ This reflects symptoms of comorbidities reported by parent at time of survey completion-not diagnosed comorbidities. The comparator for each comorbidity group was no comorbidity. Many children screened positive for more than one comorbidity.

study period had 1.5 times greater odds of sharing (OR, 1.52 [95\% CI: 1.03, 2.23]. Finally, parents tended to be more likely to share an initial compared with a follow-up survey (OR,
1.61 [95\% CI: 0.97, 2.66], $p=0.06)$. In these models, prior diagnosis of ADHD and ADHD symptom scores were not associated with information sharing. 
Table 3 Multivariable associations of ADHD characteristics with parents' decision to share ADHD survey information with teachers at the first opportunity

\begin{tabular}{|l|l|}
\hline $\begin{array}{l}\text { Child's ADHD } \\
\text { characteristics }\end{array}$ & $\begin{array}{l}\text { Parent shared with teacher } \\
\text { at first opportunity, } \\
\text { odds ratio }(95 \% \mathrm{CI})^{\mathbf{a}}\end{array}$ \\
\hline $\begin{array}{l}\text { Survey type - Initial } \\
\text { (vs. follow-up) }\end{array}$ & $1.61(0.97,2.66)$ \\
\hline $\begin{array}{l}\text { Teacher completed a survey } \\
\text { during study (vs. not) }\end{array}$ & $1.52(1.03,2.23)$ \\
\hline $\begin{array}{l}\text { ADHD diagnosis or problem } \\
\text { list entry }\end{array}$ & $0.79(0.40,1.59)$ \\
\hline $\begin{array}{l}\text { ADHD symptoms at time } \\
\text { of survey completion }\end{array}$ & Reference \\
\hline No symptoms & $1.12(0.67,1.88)$ \\
\hline Inattentiveness only & $0.92(0.40,2.14)$ \\
\hline Hyperactivity only & $0.88(0.52,1.50)$ \\
\hline Combined symptoms & Reference \\
\hline $\begin{array}{l}\text { ADHD-related performance } \\
\text { impairments }\end{array}$ & $1.95(1.21,3.15)$ \\
\hline None & $2.03(1.09,3.76)$ \\
\hline $\begin{array}{l}\text { Academic impairment } \\
\text { only }\end{array}$ & $\begin{array}{l}\text { Interpersonal impairment } \\
\text { only }\end{array}$ \\
\hline $\begin{array}{l}\text { Both academic and } \\
\text { interpersonal impairments }\end{array}$ & $1.26,3.44)$ \\
\hline
\end{tabular}

Abbreviations: ADHD, attention-deficit/hyperactivity disorder; $\mathrm{Cl}$, confidence interval; EHR, electronic health record; OR, odds ratio. ${ }^{a}$ Results are from multivariable logistic regression of the characteristics above and sharing at the first opportunity. ADHD characteristics that were included were bivariately associated with sharing $(p<0.1$ as cutoff). Models additionally adjusted for patient demographic and clinical characteristics that were bivariately associated with sharing, including child age, race, insurance payer, and ADHD medication use (documented in the EHR or based on parent report).

\section{Viewing of Surveys}

Of the 667 shared parent surveys, 107 (16.0\%) were viewed by the child's teacher, whereas 149 of the 493 teacher surveys were viewed by parents (30.2\%). Parents and teachers viewed their own survey results at similar rates: 71 out of 1,006 total parent surveys (7.1\%) and 38 out of 493 total teacher surveys (7.7\%) (chi-square, $p=0.2$ ). Parents and teachers were both more likely to view information provided by the other rather than viewing their own results. There was no relationship between ADHD diagnosis and either parent or teacher viewing email results. Teachers of children in the suburban setting and of children with private insurance were significantly more likely to view shared surveys ( $8.8 \%$ vs. $20.3 \%$ [ $p<0.001]$ and $19.6 \%$ vs. $19.9 \%$ [ $p=0.001$ ], respectively).

\section{Subsequent Sharing}

A total of 152 parents/guardians shared survey results with teachers on the first opportunity and completed at least two surveys during the study period (25.8\% of the total sample). Among those with the opportunity to share again, 135 (88.8\%) shared results with teachers on a second occasion. There was no difference in the percent of parents viewing teacher surveys between those who did and did not share a later survey (17.0\% vs. 17.7\%). In addition, 22 (30.9\%) parents/ guardians who elected not to share at the first opportunity did choose to share at a subsequent opportunity.

\section{Discussion}

Fragmentation of services may lead to care inefficiencies and suboptimal care. ${ }^{15}$ Technology can help promote SDM and decrease fragmentation but has not been widely integrated in ADHD management. ${ }^{40}$ SDM in ADHD depends upon information exchange among parents, clinicians, and teachers, yet multiple barriers to this process remain. Our results indicate that the majority of families were willing to share relevant health information with teachers using a technology-assisted approach, which can promote SDM. Performance impairments $(\mathrm{OR}, 2.09)$ were the strongest patient characteristic associated with sharing, with younger patient age also noted to be an important predictor, consistent with other literature on ADHD help-seeking adolescents. ${ }^{41,42}$ Younger patient age was also noted to be an important predictor which may be reflective of a trend for parents to become less involved in their children's schooling when they become adolescents. ${ }^{43,44}$ This is consistent with other studies addressing barriers to ADHD treatment. ${ }^{20}$ Despite a large quantity of sharing, only a minority of shared surveys were viewed; parents and teachers viewed 16 and $30 \%$ of shared surveys, respectively.

Our results indicate that the majority of families are willing to share health information with teachers when that shared information is essential to effective treatment. Despite good reasons for having privacy laws (HIPAA for health care and FERPA for scholastic areas), these regulatory burdens can impair the sharing of information, even when all stakeholders agree that sharing is desirable. Our ADHD information sharing system, which adhered to all regulatory constraints, demonstrated that a technological approach to sharing is acceptable to families. Despite needing to opt in for sharing, most parents (64.4\%) decided to share at least part of their survey responses with their child's teacher and $80 \%$ who elected to share, shared everything. We also found that parents who started using the system after the sharing features were activated (i.e., families completing initial Vanderbilt surveys with the new system) tended to share more than established users of the system (i.e., parents completing follow-up surveys when sharing features became available). This could reflect that the novelty of the system was a driving force for sharing. ${ }^{4,46}$ Sharing rates for initial surveys may also be higher for other reasons, including potentially the ability to share the treatment goal section, which is unique to the initial survey. Those parents who elected to share on the initial survey were very likely to share subsequent surveys suggesting a perceived benefit for parents from sharing, given the need to opt in at each opportunity. ${ }^{47}$ Other factors that could have influenced sharing include parental trust in the medical system and the influence of the 
primary care physician on the parent decision-making process, even outside of the office visit.

We hypothesized that increased ADHD severity would be associated with increased sharing. Interestingly, we found performance impairments were associated with sharing after controlling for confounding, but simply having symptoms was not. This finding likely reflects the perceived importance of performance (e.g., academic problems) as opposed to symptoms in seeking to enlist a teacher's help by sharing information. ${ }^{41,42,48,49}$ Parents also could be more interested in sharing if they believed that there was something that needed to be fixed. ${ }^{31,32,41,42}$ Our results suggest the need to especially encourage system use among families who most need support to address these impairments, as they are those most likely to utilize the tools.

Although sharing was common, our results indicate a need for developing improved strategies to support viewing of shared results. Despite only $16 \%$ of parents and $30 \%$ of teachers cross-reviewing submitted surveys, we were encouraged by these rates of viewing given numerous barriers to accessing messages through the secure messaging software. ${ }^{39}$ These findings suggest that some families and teachers see more value in reviewing the information than others. There is known variation in patient engagement and adherence to recommendations in families of children with ADHD. ${ }^{20}$ We could potentially improve sharing and viewing by highlighting these features during patient registration, providing support for clinicians, onboarding families, and facilitating teacher enrollment.

Within our system, viewing surveys required parents and teachers to access information through a secure email delivery service, which required its own logon and password. This process was thought to be more difficult than simply electing to share via a survey question. Simplifying access to this system using our institution's personal health record, providing logon support, the creation of a mobilefriendly portal, and/or allowing teachers restricted access to a patient's EHR portal could improve viewing of shared information. Evidence-based strategies could also be employed to encourage the viewing of shared information including sending electronic reminders or the use of incentives. ${ }^{50,51}$ Teachers of children in suburban practices and on private insurance were more likely to view information, potentially indicating that socioeconomic status of the child or resources of the school system play a role in teachers viewing information. From stakeholders, we learned that urban teachers may be responsible for large numbers of students, with diverse educational needs, and time constraints alone may have limited viewing. Additional supports may be needed to help teachers in the urban setting and/or who provide education for children with public insurance in accessing and viewing the shared surveys. Finally, the emails only provided the data and data trends without guidance for parents or teachers on what to do next. Parents and teachers may have been less likely to open emails if they did not have a clear action to take based on the information provided. Future revisions to the tool may best support families if suggested actions are highlighted. It is also possible that the parents elected to share information with teachers without informing the teachers. In these circumstances, it would be entirely likely that a teacher would not view the email.

Many children with ADHD are managed by external providers, and clinicians caring for these patients would be unlikely to utilize the ADHD Care Assistant for these families, which helps to explain why only $14 \%$ of identified patients were registered for the system. In addition, certain clinicians and practices had existing established workflows for ADHD and were reluctant to change their processes by using the electronic system. This study had several limitations. The patients in this study represented a limited subset of all patients seen in our practices with ADHD and for whom screening for ADHD was triggered by the EHR. It may be difficult to draw generalizations to the larger population, especially those with ADHD managed outside of primary care or whose parents are less comfortable using technology to manage health concerns. The focus of the evaluation was selection of sharing for parents who already opted in to use the system. Parents who elected to use this system may be different from parents who opted not to use this system and why parents and teachers elected to complete or not complete a survey was beyond the scope of this study. An evaluation of the factors that influence use of this type of system will require future study. From a design standpoint, the use of observational data limits our ability to establish causation, and therefore it is difficult to identify precisely those factors that can improve sharing. We had limited control over when and why clinicians chose to use the system, resulting in activation of the system for children less than 5 years old. Given the small number of preschoolers included, it would be difficult to draw conclusions from this study for this population. We also lacked information regarding clinician, parent, and teacher demographics so we cannot draw conclusions related to nonpatient demographics. In addition, our reliance on technology may limit the generalizability of the approaches we used in health systems with older or more constrained EHRs, which are common in many practice settings. Nonetheless, Web services like Health Level-7's Fast Healthcare Interoperability Resources and our own Care Assistant framework are increasingly common with many EHR vendors and are likely to increasingly support the implementation of novel informatics approach to care, such as the partnership between families and teachers described in this article. As with many CDS interventions, sustainability and dissemination are concerns. We have transitioned ownership of this project from a research team to our operational team and hope to generate lessons learned about this process and to support dissemination beyond our organizational walls. Future studies involving this intervention will address how sharing of information impacts ADHD-related outcomes (i.e., symptoms, performance impairment, and medication use), the value of sharing ADHD scores between parents and teachers, whether we can decrease barriers to sharing of information, and whether this approach can be applied to other disorders affecting health and school performance. 


\section{Conclusion}

Electronic tools to support communication can decrease fragmentation of care and support SDM for children with ADHD. Most parents were willing to share ADHD-related information with teachers, and those with more severely affected children were most likely to share information. Despite barriers to accessing information, parents and teachers successfully viewed the shared surveys at modest rates. System enhancements to facilitate the viewing of shared surveys are technologically feasible and should be explored in future studies. Future work is needed to determine if sharing information impacts patient care outcomes relevant to ADHD.

\section{Clinical Relevance Statement}

Treatment for children with ADHD is often fragmented leading to suboptimal care. Electronic tools may foster communication but have not yet been applied for improving communication among parents, teachers, and clinicians during the provision of ADHD management. We demonstrate the potential of using electronic systems provided by the medical home to promote parental sharing of information about their child's ADHD with teachers.

\section{Multiple Choice Questions}

1. Sharing of results and viewing of results were the two new functions developed and evaluated as part of the update to this intervention. Which of the following correctly match a clinical or patient characteristic with increased utilization of the new function?

a. Survey Type AND Sharing of Surveys by Parents.

b. Survey Type AND Viewing of Surveys by Teachers.

c. Suburban Office Setting AND Sharing of Surveys by Parents.

d. Suburban Office Setting AND Viewing of Surveys by Teachers.

e. Performance Impairments AND Sharing of Survey by Teachers.

Correct Answer: The correct answer is option d. This question addresses the evaluation of results presented using bivariate and covariate analysis. In bivariate analyses, potential relationships between patient characteristics may appear significant, but upon a covariate analysis, after controlling for patient demographics, these relationships may be found to no longer be significant. Answer a is only an association identified during the bivariate analyses. When controlling for patient characteristics, it was not found to be significant. Additionally, the question asks for a clinical or patient characteristic and this is an ADHD survey characteristic. Answer b is incorrect as viewing of surveys by teachers was not investigated for different survey types (insufficient power to perform this analysis). Answer c is incorrect as suburban office setting was found to be unrelated to sharing in the bivariate analysis. Answer d reports a correct association of a patient characteristic and a significant finding. Answer e is incorrect because it is sharing by parents and not sharing by teachers that was investigated.

2. Multiple potential barriers were identified to parent and teacher viewing of survey results. Which follow-up study could be performed to evaluate a potential solution to a technologic barrier of viewing emailed surveys?

a. Repeating the study with an increase in sample size to overcome barriers to viewing.

b. Randomizing parents to receive secure versus nonsecure emails with ADHD survey results.

c. Switching from an email-based system to a Web portalbased system for accessing records.

d. Compensating families and teachers each time they viewed a new survey result.

e. Allowing multiple teachers to have access to the system to increase the likelihood of a tech-savvy participant.

Correct Answer: The correct answer is option b. This question addresses different ways future studies could be performed to address technical limitations of this study. Answer b directly addresses the proposed technical limitation that SEND SECURE messages deter people from opening for results. Answer a would not address the technical barrier, but could be useful in determining if patient characteristics are related to viewing by increasing the study power. Answer c would potentially increase views but would not directly target the technical barrier of needing to have a login and password to obtain the results. Compensating families (answer d) might improve rates but would not address the technical barriers. Answer e would also potentially increase views but would not address the technical barriers.

\section{Protection of Human and Animal Subjects}

This study was performed in compliance with the World Medical Association Declaration of Helsinki on Ethical Principles for Medical Research Involving Human Subjects, and was reviewed by the CHOP's Institutional Review Board.

\section{Funding}

This work was funded through the Patient Centered Outcomes Research Institute (CDR-1408-20669).

\section{Conflict of Interest}

Dr. Michel, Dr. Grundmeier, and Mr. Miller are the primary authors of the ADHD Care Assistant, which was used to implement portions of the intervention evaluated in this article. No patent or licensing agreement exists for this technology and the invention has generated no revenue. The remaining authors have no conflicts of interest relevant to this article to disclose.

Drs. Grundmeier and Fiks are coinventors of the Care Assistant framework, which was used to implement portions of the intervention evaluated in this article. No patent or licensing agreement exists for this technology and the invention has generated no revenue. The remaining authors have no financial relationships relevant to this article to 
disclose. Though he personally took no salary support, Dr. Fiks's research team received grant support from Pfizer for an independent research project to improve ADHD care.

\section{Acknowledgments}

We thank the network of primary care physicians and their patients and families for their contributions to clinical research through the $\mathrm{CHOP}$ ambulatory care network including involvement in the stakeholder workgroups which informed the updates to the ADHD Care Assistant. We wish to acknowledge Jeritt Thayer and Dean Karavite for their contributions to development and deployment of the ADHD Care Assistant.

\section{References}

1 Sharma A, Couture J. A review of the pathophysiology, etiology, and treatment of attention-deficit hyperactivity disorder (ADHD). Ann Pharmacother 2014;48(02):209-225

2 Wolraich M, Brown L, Brown RT, et al; Subcommittee on Attention-Deficit/Hyperactivity Disorder; Steering Committee on Quality Improvement and Management. ADHD: clinical practice guideline for the diagnosis, evaluation, and treatment of attention-deficit/hyperactivity disorder in children and adolescents. Pediatrics 2011;128(05):1007-1022

3 Polanczyk GV, Willcutt EG, Salum GA, Kieling C, Rohde LA. ADHD prevalence estimates across three decades: an updated systematic review and meta-regression analysis. Int J Epidemiol 2014;43 (02):434-442

4 Wolraich ML, Feurer ID, Hannah JN, Baumgaertel A, Pinnock TY. Obtaining systematic teacher reports of disruptive behavior disorders utilizing DSM-IV.J Abnorm Child Psychol 1998;26(02):141-152

5 Murray E, Charles C, Gafni A. Shared decision-making in primary care: tailoring the Charles et al. model to fit the context of general practice. Patient Educ Couns 2006;62(02):205-211

6 Fiks AG, Mayne S, Debartolo E, Power TJ, Guevara JP. Parental preferences and goals regarding ADHD treatment. Pediatrics 2013;132(04):692-702

7 Brinkman WB, Hartl Majcher J, Poling LM, et al. Shared decisionmaking to improve attention-deficit hyperactivity disorder care. Patient Educ Couns 2013;93(01):95-101

8 Lyon AR, Whitaker K, French WP, Richardson LP, Wasse JK, McCauley E. Collaborative care in schools: enhancing integration and impact in youth mental health. Adv Sch Ment Health Promot 2016;9(3-4):148-168

9 Hoagwood K, Kelleher KJ, Feil M, Comer DM. Treatment services for children with ADHD: a national perspective. J Am Acad Child Adolesc Psychiatry 2000;39(02):198-206

10 Zwi M, Jones H, Thorgaard C, York A, Dennis JA. Parent training interventions for attention deficit hyperactivity disorder (ADHD) in children aged 5 to 18 years. Cochrane Database Syst Rev 2011; (12):CD003018

11 DuPaul GJ, Gormley MJ, Laracy SD. School-based interventions for elementary school students with ADHD. Child Adolesc Psychiatr Clin N Am 2014;23(04):687-697

12 Lange AM, Daley D, Frydenberg M, Rask CU, Sonuga-Barke E, Thomsen PH. The effectiveness of parent training as a treatment for preschool attention-deficit/hyperactivity disorder: study protocol for a randomized controlled, multicenter trial of the New Forest Parenting Program in Everyday Clinical Practice. JMIR Res Protoc 2016;5(02):e51

13 Wagner EH. The role of patient care teams in chronic disease management. BMJ 2000;320(7234):569-572

14 Bilgrami Z, McLaughlin L, Milanaik R, Adesman A. Health implications of new-age technologies: a systematic review. Minerva Pediatr 2017;69(04):348-367
15 Guevara JP, Feudtner C, Romer D, et al. Fragmented care for innercity minority children with attention-deficit/hyperactivity disorder. Pediatrics 2005;116(04):e512-e517

16 Wolraich ML, Bickman L, Lambert EW, Simmons T, Doffing MA. Intervening to improve communication between parents, teachers, and primary care providers of children with ADHD or at high risk for ADHD. J Atten Disord 2005;9(01):354-368

17 Power TJ, Blum NJ, Guevara JP, Jones HA, Leslie LK. Coordinating mental health care across primary care and schools: ADHD as a case example. Adv Sch Ment Health Promot 2013;6(01): $68-80$

18 Reinke WM, Stormont M, Herman KC, Puri R, Goel N. Supporting children's mental health in schools: teacher perceptions of needs, roles, and barriers. Sch Psychol Q 2011;26(01):1-13

19 Heuer B, Williams S. Collaboration between PNPs and school nurses: meeting the complex medical and academic needs of the child with ADHD. J Pediatr Health Care 2016;30(01):88-93

20 Corkum P, Bessey M, McGonnell M, Dorbeck A. Barriers to evidencebased treatment for children with attention-deficit/hyperactivity disorder. Atten Defic Hyperact Disord 2015;7(01):49-74

21 Wolraich ML, Lambert W, Doffing MA, Bickman L, Simmons T, Worley K. Psychometric properties of the Vanderbilt ADHD diagnostic parent rating scale in a referred population. J Pediatr Psychol 2003;28(08):559-567

22 Chang LY, Wang MY, Tsai PS. Diagnostic accuracy of rating scales for attention-deficit/hyperactivity disorder: a meta-analysis. Pediatrics 2016;137(03):e20152749

23 Brinkman WB, Epstein JN. Promoting productive interactions between parents and physicians in the treatment of children with attention-deficit/hyperactivity disorder. Expert Rev Neurother 2011;11(04):579-588

24 Hart CN, Kelleher KJ, Drotar D, Scholle SH. Parent-provider communication and parental satisfaction with care of children with psychosocial problems. Patient Educ Couns 2007;68(02): 179-185

25 Patel A, Medhekar R, Ochoa-Perez M, et al. Care provision and prescribing practices of physicians treating children and adolescents with ADHD. Psychiatr Serv 2017;68(07):681-688

26 Power TJ, Michel J, Mayne S, et al. Coordinating systems of care using health information technology: development of the ADHD care assistant. Adv Sch Ment Health Promot 2016;9(3-4):201-218

27 Fiks AG, Grundmeier RW, Biggs LM, Localio AR, Alessandrini EA. Impact of clinical alerts within an electronic health record on routine childhood immunization in an urban pediatric population. Pediatrics 2007;120(04):707-714

28 Fiks AG, Zhang P, Localio AR, et al. Adoption of electronic medical record-based decision support for otitis media in children. Health Serv Res 2015;50(02):489-513

29 Tham E, Swietlik M, Deakyne S, et al; Pediatric Emergency Care Applied Research Network (PECARN). Clinical decision support for a multicenter trial of pediatric head trauma: development, implementation, and lessons learned. Appl Clin Inform 2016;7(02):534-542

30 Michel J, Utidjian LH, Karavite D, et al. Rapid adjustment of clinical decision support in response to updated recommendations for palivizumab eligibility. Appl Clin Inform 2017;8(02):581-592

31 Fiks AG, Mayne S, Karavite DJ, DeBartolo E, Grundmeier RW. A shared e-decision support portal for pediatric asthma. J Ambul Care Manage 2014;37(02):120-126

32 Fiks AG, Mayne SL, Karavite DJ, et al. Parent-reported outcomes of a shared decision-making portal in asthma: a practice-based RCT. Pediatrics 2015;135(04):e965-e973

33 Epstein JN, Langberg JM, Lichtenstein PK, Kolb R, Simon JO. The myADHDportal.com Improvement Program: an innovative quality improvement intervention for improving the quality of ADHD care among community-based pediatricians. Clin Pract Pediatr Psychol 2013;1(01):55-67

34 Epstein JN, Langberg JM, Lichtenstein PK, Kolb R, Altaye M, Simon JO. Use of an Internet portal to improve community-based 
pediatric ADHD care: a cluster randomized trial. Pediatrics 2011; 128(05):e1201-e1208

35 Epstein JN, Kelleher KJ, Baum R, et al. Impact of a web-portal intervention on community ADHD care and outcomes. Pediatrics 2016;138(02):e20154240

36 Baum RA, Epstein JN, Kelleher K. Healthcare reform, quality, and technology: ADHD as a case study. Curr Psychiatry Rep 2013;15 (07):369

37 Fiks AG, Mayne SL, Michel JJ, et al. Distance-learning, ADHD quality improvement in primary care: a cluster-randomized trial. J Dev Behav Pediatr 2017;38(08):573-583

38 Fiks AG, Mayne S, Hughes CC, et al. Development of an instrument to measure parents' preferences and goals for the treatment of attention deficit-hyperactivity disorder. Acad Pediatr 2012;12 (05):445-455

39 Cisco Registered Envelope Service. San Jose, CA: Cisco Systems, INC.; 2017

40 Schatz NK, Fabiano GA, Cunningham CE, et al. Systematic review of patients' and parents' preferences for ADHD treatment options and processes of care. Patient 2015;8(06):483-497

41 Eiraldi RB, Mazzuca LB, Clarke AT, Power TJ. Service utilization among ethnic minority children with ADHD: a model of helpseeking behavior. Adm Policy Ment Health 2006;33(05):607-622

42 Bussing R, Zima BT, Gary FA, Garvan CW. Barriers to detection, help-seeking, and service use for children with ADHD symptoms. J Behav Health Serv Res 2003;30(02):176-189
43 Child Trends. Parental involvement in schools; 2013. Available at: https://www.childtrends.org/?indicators=parental-involvementin-schools. Accessed November 29, 2018

44 Hill N, Taylor L. Parental school involvement and children's academic achievement: pragmatics and issues. Curr Dir Psychol Sci 2004;13(04):161-164

45 Gardner B, Phillips LA, Judah G. Habitual instigation and habitual execution: definition, measurement, and effects on behaviour frequency. Br J Health Psychol 2016;21(03):613-630

46 Schomaker J, Meeter M. Short- and long-lasting consequences of novelty, deviance and surprise on brain and cognition. Neurosci Biobehav Rev 2015;55:268-279

47 Kwahk K-Y, Ahn H, Ryu YU. Understanding mandatory IS use behavior: how outcome expectations affect conative IS use. Int J Inf Manage 2018;38(01):64-76

48 Gulliver A, Griffiths KM, Christensen H. Perceived barriers and facilitators to mental health help-seeking in young people: a systematic review. BMC Psychiatry 2010;10(01):113

49 Rickwood DJ, Deane FP, Wilson CJ. When and how do young people seek professional help for mental health problems? Med J Aust 2007;187(7, Suppl):S35-S39

50 Tao D, Xie L, Wang T, Wang T. A meta-analysis of the use of electronic reminders for patient adherence to medication in chronic disease care. J Telemed Telecare 2015;21(01):3-13

51 Coleman MT, Pasternak RH. Effective strategies for behavior change. Prim Care 2012;39(02):281-305 